\title{
Kinesin-1 and Dynein Are the Primary Motors for Fast Transport of Mitochondria in Drosophila Motor Axons $\square$
}

\author{
Aaron D. Pilling,* Dai Horiuchi, Curtis M. Lively, and William M. Saxton
}

Department of Biology, Indiana University, Bloomington, IN 47405-3700

Submitted June 13, 2005; Accepted January 26, 2006

Monitoring Editor: J. Richard McIntosh

To address questions about mechanisms of filament-based organelle transport, a system was developed to image and track mitochondria in an intact Drosophila nervous system. Mutant analyses suggest that the primary motors for mitochondrial movement in larval motor axons are kinesin-1 (anterograde) and cytoplasmic dynein (retrograde), and interestingly that kinesin-1 is critical for retrograde transport by dynein. During transport, there was little evidence that force production by the two opposing motors was competitive, suggesting a mechanism for alternate coordination. Tests of the possible coordination factor $\mathrm{P} 150$ Glued suggested that it indeed influenced both motors on axonal mitochondria, but there was no evidence that its function was critical for the motor coordination mechanism. Observation of organelle-filled axonal swellings ("organelle jams" or "clogs") caused by kinesin and dynein mutations showed that mitochondria could move vigorously within and pass through them, indicating that they were not the simple steric transport blockades suggested previously. We speculate that axonal swellings may instead reflect sites of autophagocytosis of senescent mitochondria that are stranded in axons by retrograde transport failure; a protective process aimed at suppressing cell death signals and neurodegeneration.

\section{INTRODUCTION}

Organelle transport is central to the organization, developmental fate, and functions of asymmetric cells. A bipolar neuron is an extreme case, with the somato-dendritic region and the axon containing different sets of proteins and organelles. In general, much of the machinery for synthesizing and recycling neuronal components is clustered near the nucleus. Because an axon, often with an axial ratio of many thousands, usually contains $>99 \%$ of the neuronal cytoplasm, active transport of new components away from the cell body and of spent components and trophic materials back toward the cell body is critical. Disruptions of axonal transport are thought to contribute to the pathologies of Alzheimer's, amyotrophic lateral sclerosis, and other neurodegenerative diseases (reviewed by Mandelkow and Mandelkow, 2002; Hirokawa and Takemura, 2005).

Long-distance organelle transport in axons is driven by motor proteins that move along parallel microtubules whose plus ends are mostly oriented toward the axon terminal (reviewed by Hollenbeck and Saxton, 2005). There are multiple types of microtubule motors in postmitotic vertebrate neurons, including kinesins that can move toward either plus- or minus-ends and at least one cytoplasmic dynein that moves toward minus-ends (Martin et al., 1999b; Hirokawa and Takemura, 2005; Hollenbeck and Saxton, 2005). Which motors bind and move which axonal components? When

This article was published online ahead of print in $M B C$ in Press (http://www.molbiolcell.org/cgi/doi/10.1091/mbc.E05-06-0526) on February 8, 2006.

$\square \square$ The online version of this article contains supplemental material at MBC Online (http:/ / www.molbiolcell.org).

* Present address: Department of Physiology, University of Pennsylvania, Philadelphia, PA 19104-6085.

Address correspondence to: William M. Saxton (bsaxton@indiana. edu). multiple types of motors bind a single cargo, do they collaborate or compete, how are they regulated, and how do their combined activities generate an optimal distribution of that type of cargo?

Axonal mitochondria are critical for the physiology of neurons and are particularly well suited for studying active transport mechanisms. They are large, readily distinguished from other organelles, and they engage in long-range movements consistent with microtubule-based transport as well as short-range movements consistent with actin-based transport (Hollenbeck and Saxton, 2005). It is evident that mitochondrial positioning mechanisms are influenced by local energy requirements. In growing axons, mitochondria actively navigate toward the growth cone where ATP consumption is high, they accumulate there, and then they disperse when growth cone activity stops (Morris and Hollenbeck, 1993). It has also been shown that moving mitochondria preferentially fill in gaps between anchored mitochondria and that they congregate at sites along an axon where specific signaling pathways are activated (Chada and Hollenbeck, 2003; Miller and Sheetz, 2004). This complex and purposeful behavior provides a rich context for answering questions about motor activities and the control of those activities by cellular cues.

To begin addressing questions about mitochondrial transport mechanisms, we used an approach that allows digital tracking of green fluorescent protein (GFP)-loaded mitochondria in motor axons of an intact Drosophila nervous system. Our results suggest a model in which mitochondria from the cell body are carried anterograde by kinesin- 1 with little interference by dynein, supporting the idea of alternating coordination of the opposing motors. Anterograde mitochondria then switch to a stationary state, presumably to satisfy local energy needs. In time, stationary mitochondria that become metabolically impaired switch from a mainly stationary to a retrograde state by activating cytoplasmic dynein to drive movement back toward the cell body (Morris and Hollenbeck, 1993; Miller and Sheetz, 2004). Surprisingly, 
retrograde transport by dynein requires kinesin-1, which supports the possibility that kinesin acts as a biochemical or biophysical activator of dynein (Martin et al., 1999a; Brendza et al., 2002; Ligon et al., 2004). We discuss the idea that failed retrograde transport of spent mitochondria stimulates axonal autophagy and axonal swellings as a means to suppress cell death signals and neurodegeneration.

\section{MATERIALS AND METHODS}

\section{Molecular Constructs and Fly Transformation}

To create a Gal4-responsive mitochondria-targeted GFP transgene, a chimeric gene encoding the N-terminal 31 amino acids of human cytochrome $c$ oxidase subunit VIII (cCoxVIII) followed by a gene encoding GFP(S65T) (Rizzuto et al., 1995) was isolated from mito-GFP(S65T)/pBSK ${ }^{+}$by digestion with EcoRI/XhoI. The N-terminal 25 amino acids of cCoxVIII act as a cleavable presequence for mitochondrial import (Rizzuto et al., 1989). The chimeric gene was ligated into the Drosophila transformation vector pUAST, downstream of a GAL4-responsive promoter to allow tissue-specific control of GFP expression (Brand and Perrimon, 1993). The resulting transposable element, $P\left[w^{+m C}=U A S-m i t o G F P . A P\right]$, was injected into $w$; e/Sb $P\left[r y^{+}, \Delta 2-3\right]$ embryos that expressed transposase. Several independent stably transformed lines were established.

A Glued RNA interference (RNAi) snapback construct was created with a 0.7-kb PCR fragment of Glued from the plasmid RE24170 (Drosophila Genomics Resource Center, Bloomington, IN). The fragment was subcloned into the $3^{\prime}$ splice site of the Drosophila RNAi vector pWIZ at the NheI site (Lee and Carthew, 2003). A complimentary fragment was subcloned into the $5^{\prime}$ splice site of pWIZ at the AvrII site. The resulting transposable element containing Glued inverted repeats $(P[p W I Z=U A S-G l u e d R N A i S n a p . A P])$ was injected into $w$; $e$ embryos with a transposase-expressing helper plasmid.

\section{Drosophila Culture and Genetics}

Flies were cultured at $25^{\circ} \mathrm{C}$ in a 12 -h light/dark cycle on standard soft medium ( $0.5 \%$ agar, $7 \%$ molasses, $6 \%$ cornmeal, and $0.8 \%$ killed yeast) seeded with live yeast. Descriptions of the mutant alleles and balancers used can be found in FlyBase (http: / / flybase.bio.indiana.edu/) and several publications (Saxton et al., 1991; Gepner et al., 1996; Martin et al., 1999a). Standard meiotic recombination approaches were used to generate third chromosomes with both a motoneuron Gal4 driver, $P[$ GawB $] D 42$ (Yeh et al., 1995), and a GFP responder $P\left[w^{+m C}=\right.$ $U A S-m i t o G F P . A P] 3$. Recombinant chromosomes were balanced over $T M 6 B(T b$ $\mathrm{Hu}$ e $\mathrm{ca}$ ). Standard strain construction approaches were used to generate heterozygous $K h c$ mutant flies that carried both driver and responder transgenes (Khc mutant $/ C y o ; P[G a w B] D 42 P\left[w^{+m C}=U A S\right.$-mitoGFP.AP $] 3 / P[G a w B] D 42 P\left[w^{+m C}=\right.$ UAS-mitoGFP.AP]3). To obtain equivalent balanced Dhc64C, Gl ${ }^{1}$, and $P[W I Z=$ UAS-GluedRNAi.AP] strains, meiotic recombination was used to generate flies that carried Dhc64C mutant $\mathrm{Gl}^{1}$, or $p[W I Z=U A S-G l u e d R N A i . A P]$ with $P[$ GawB $] D 42 P\left[w^{+m C}=U A S-m i t o G F P . A P\right] 3$.

\section{Giant Cultured Neurons and Larval Immunostaining}

Cells were dissociated from $w ; P[$ GawB $] D 42 P\left[w^{+m C}=U A S-m i t o G F P . A P\right] 3$ embryos $4 \mathrm{~h}$ after fertilization and then cultured on glass coverslips in the presence of $2 \mu \mathrm{g} / \mathrm{ml}$ cytochalasin. This generated large polyploid cells that differentiated into muscle and other cell types, including neurons with long neuritic extensions (Wu et al., 1990). Because GFP expression was driven by D42, neurons could be identified by the expression of mitochondrial GFP. Overnight cultures were incubated for $30 \mathrm{~min}$ at $20-23^{\circ} \mathrm{C}$ in modified Schneider's insect medium (Sigma-Aldrich, St. Louis, MO) containing $250 \mathrm{nM}$ MitoTracker Red CMXRos (Molecular Probes, Eugene, OR). Fixation was done for $15 \mathrm{~min}$ in Schneider's medium containing 3.7\% paraformaldehyde. Coverslips were washed four times in phosphate-buffered saline and mounted on glass slides with $90 \%$ glycerol.

To localize Khc and Dhc67C in segmental nerves, wandering third instars were dissected and prepared as described previously (Hurd and Saxton, 1996). The primary antibodies used were mouse monoclonal anti-Drosophila Dhc64C at 1:100 (Sharp et al., 2000) and affinity-purified rabbit anti-Drosophila Khc (AKIN01; Cytoskeleton, Denver, CO) at 1:100. Secondary antibodies used were affinity-purified tetramethylrhodamine B isothiocyanate-conjugated goat anti-mouse IgG $(\mathrm{H}+\mathrm{L})$ at 1:500 and affinity-purified Cy5-conjugated goat anti-rabbit $\operatorname{IgG}(\mathrm{H}+\mathrm{L})$ at 1:500 in phosphate-buffered saline/Tween (Jackson ImmunoResearch Laboratories, West Grove, PA)

Imaging of fixed/stained cells and segmental nerves was done with a PerkinElmer UltraVIEW LCI Spinning Disk confocal system on a Nikon Eclipse TE200 microscope equipped with a Nikon 100× Planapo objective (numerical aperture [NA] 1.40). Images were processed in NIH Image version $1.62 \mathrm{~b} 7$ (National Institutes of Health, Bethesda, MD) and Adobe Photoshop version 7.0 (Adobe Systems, Mountain View, CA).

\section{Time-Lapse Microscopy of Mitochondria}

To image mitochondria in live cultured neurites, cells grown on coverslips were placed over the well of a 2-ml Pyrex depression slide. Fresh Schneider's medium was perfused in every few minutes. Cells with GFP-mitochondria were imaged with an MRC600 laser scanning confocal fluorescence system (Bio-Rad, Hercules, CA) attached to a Nikon Optiphot microscope equipped with a Nikon $60 \times$ objective (NA 1.40). Images were acquired at a rate of one frame every $2.2 \mathrm{~s}$ for $220 \mathrm{~s}$. Movement of GFP-mitochondria was active for long periods. However, to minimize physiological variability, data were collected within $1 \mathrm{~h}$ of mounting.

To image mitochondria in live segmental nerves, third instars were dissected quickly (<5min) in Schneider's medium (Hurd and Saxton, 1996). The resulting neuromuscular preparations were spread on a glass coverslip with their internal sides against the glass, mounted over a depression slide with medium, perfused as described above, and viewed with the MRC600 confocal at room temperature $\left(\sim 22^{\circ} \mathrm{C}\right)$, as described above.

To allow accurate long-range tracking of mitochondrial transport, a 55- $\mu \mathrm{m}$ region of the segmental nerve was photobleached for $15 \mathrm{~s}$ with 488-nm light at full intensity from the MRC600 confocal laser (60× objective, zoom factor 4). Immediately after photobleaching, images were collected at a rate of 1 frame/s (zoom factor 2.5) for $300 \mathrm{~s}$. The confocal output was coupled directly to the video input of an Apple Macintosh Power PC 8500/150 equipped with a Scion AG-5 frame grabber video card. Images were acquired using NIH Image version $1.62 \mathrm{~b} 7$ software. With frequent perfusion of culture medium, active transport of mitochondria in segmental nerves could be maintained for up to $2 \mathrm{~h}$ after dissection. However, to minimize physiological variability, recordings were collected from specimens only within $20 \mathrm{~min}$ of the start of dissection, and only one time-lapse recording was acquired from each larva. Comparisons of mitochondrial movement in bleached versus unbleached nerves did not reveal significant differences, suggesting that the photobleaching routine used did not alter mitochondrial transport.

\section{Organelle Tracking and Data Analysis}

To mark the positions of individual GFP-mitochondria, NIH Image version $1.62 \mathrm{~b} 7$ was used with an object tracking macro based on software designed by Kurt Anderson and Rob Cross and modified by A. Pilling. To minimize bias in the selection of GFP-mitochondria for tracking in cultured cell neurites and motor axons, all clearly imaged mitochondria that moved were analyzed. A mouse-driven cursor was used to generate position data by marking the center of a fluorescent mitochondrion in every consecutive confocal frame in which it was visible. The distance moved between consecutive frames was converted from pixels to real measurements based on calibration of the $x$ - and $y$-axes with a stage micrometer. For the MRC600 confocal used, the $x$ and $y$ pixel dimensions were 108 and $95 \mathrm{~nm}$, respectively.

To determine the accuracy of the marking system, nerves in a fixed larva were imaged in time-lapse and the positions of 10 mitochondria were marked in each frame. Mean displacement between frames, which should reflect variation in judging the center of each mitochondrion plus stage drift, was $0.017 \pm 0.065 \mu \mathrm{m}(\mathrm{n}=2990$ measurements $)$. A normal distribution calculated with Z-scores corresponding to $95 \%$ of the tracking error distribution ( $\pm 1 \mathrm{SD}$ ) gave an interval score from 0.11 to $0.14 \mu \mathrm{m}$. From this, the probability of an organelle displacement of $0.1 \mu \mathrm{m}$ ( $\sim 1$ pixel) between consecutive frames being due to tracking error or stage drift was $<2.4 \%$.

To define the direction of motion, mitochondria displacements were modeled as a two state binary system, consisting of plus-end (anterograde) or minus-end (retrograde) movements in the x-coordinate plane. Before each recording, the neuroblast or segmental nerve was oriented with its major axis of displacement along the x-coordinate plane, and cell body to the left. Anterograde displacements (right) were given a positive designation and retrograde displacements (left) were given a negative designation.

To describe the transport behavior of GFP-mitochondria in detail and to compare the effects of mutations on transport parameters, the chronological records of mitochondrial motility were modeled as a three state system consisting of plus-end runs, minus-end runs, and pauses, based on periods of uninterrupted motion for runs or lack of motion for pauses. Because the minimum measurable position change between consecutive frames was 0.10 $\mu \mathrm{m}$ (1 pixel), only movements with average velocities greater than $0.10 \mu \mathrm{m} / \mathrm{s}$ or less than $-0.10 \mu \mathrm{m} / \mathrm{s}$ for at least three consecutive frames were designated as runs. Periods with average velocities between 0.10 and $-0.10 \mu \mathrm{m} / \mathrm{s}$ for at least two consecutive frames were designated as pauses. A run could be preceded and ended by pauses or by oppositely-directed runs. Subsecond pauses and runs at low velocities almost certainly occur, so our boundaries between different states provide an approximation of the true run-pause behavior of mitochondria; an approximation useful for comparing the effects of experimental variables, such as genotype. After sorting each mitochondrial transport record into the three states, transport parameters were calculated: plus- and minus-end run velocities, run durations, run lengths, and pause durations. The "duty cycles" of transport represent the percentage of time mitochondria spent in each of plus-end runs, minus-end runs, and pauses.

To determine whether a single transport variable was significantly different due to genotype, linear contrast algorithms were developed and used. Because the variances of run and pause parameters for individual mitochondria 
Figure 1. Fluorescence pattern of mitoGFP expressed by the $D 42$ driver. (A) A composite image of a fixed third instar neuromuscular preparation from a larva homozygous for both the D42 Gal4 driver and mitoGFP. GFP fluorescence was strong in the presumptive optic lobes and ventral ganglion (VG), segmental nerves (SN), and neuromuscular junctions (NMJ). In this specimen, the ventral ganglion is lying on its side with optic lobes projecting down. Abdominal segments A2 and A6 are indicated. (B) A high-magnification view of a portion of a single segmental nerve passing through segment A4. Single axonal mitochondria usually were elongated (arrow) but some were small and spherical. Anterior is to the left in this, and all subsequent figures.

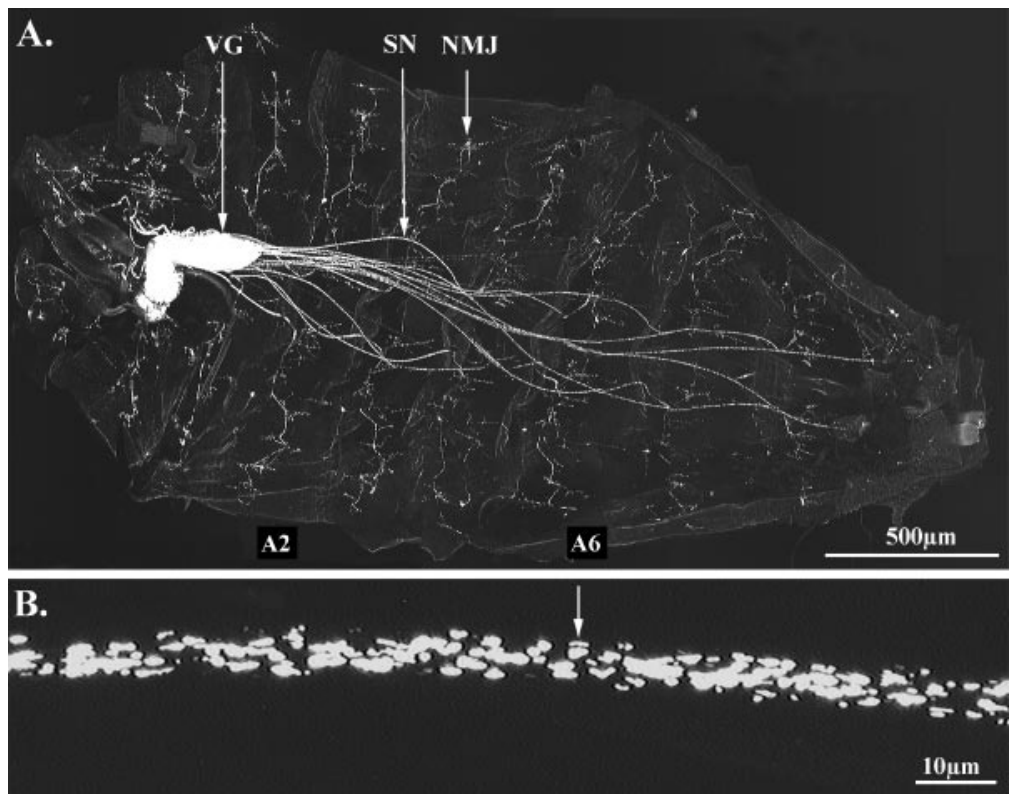

were small, to avoid repeated measures, motility parameters for different runs and pauses were aggregated for each mitochondrion. Because the variances for those aggregate parameters were large between mitochondria of an individual larva, motility parameters were not aggregated for mitochondria within a larva. Instead, the means for the individual mitochondria for each larva within a mutant genotype were compared with those of wild-type. Linear contrast analysis, using this genotype-by-larva approach, prevented larvae with large variances or sample sizes from contributing disproportionately to a single genotype mean. This also ensured smaller degrees of freedom, resulting in more stringent statistical contrasts. Motility parameters showing a genotype-by-larva contrast significance of $<0.05$ were accepted as significantly different. Equal variances were not assumed. Graphing, modeling, and statistical analysis of organelle tracking data were done using SPSS Base 10.0 (SPSS, Chicago, IL) and Microsoft Excel (Microsoft, Redmond, WA).

\section{Fractionation of Mitochondria and Western Blotting}

To fractionate Drosophila tissues, $1 \mathrm{~g}$ of adult $P[$ GawB $] D 42 P\left[w^{+m C}=U A S\right.$ mitoGFP.AP]3 flies was collected and homogenized using a mortar and pestle in $10 \mathrm{ml}$ of homogenization buffer $(250 \mathrm{mM}$ sucrose, $20 \mathrm{mM}$ HEPES, $100 \mathrm{mM}$ K-acetate, $40 \mathrm{mM} \mathrm{KCl}, 5 \mathrm{mM}$ EGTA, $5 \mathrm{mM} \mathrm{MgCl}$, 2 mM MgATP, pH 7.4 with $\mathrm{KOH}$, and one tablet of Complete protease inhibitors [Roche Diagnostics, Indianapolis, IN]). Crude homogenate was centrifuged at low speed for $3 \mathrm{~min}$ at $4^{\circ} \mathrm{C}$ in a benchtop centrifuge $(550 \times g)$ to pellet debris and nuclei. Supernatant was collected and centrifuged for $5 \mathrm{~min}$ at $4^{\circ} \mathrm{C}(5500 \times \mathrm{g})$ to pellet intact mitochondria. That "P2" pellet was gently resuspended in the original volume of homogenization buffer. To separate mitochondria from other organelles, $1 \mathrm{ml}$ of resuspended P2 was loaded on a 10-ml sucrose step gradient $(1,1.25,1.50,1.75$, and $2.00 \mathrm{M})$ in mitochondria homogenization buffer (250 $\mathrm{mM}$ sucrose, $25 \mathrm{mM}$ HEPES, $1 \mathrm{mM}$ EDTA, and $2 \mathrm{mM} \mathrm{MgCl} 2, \mathrm{pH} 7.2$ with $\mathrm{KOH}$ ) without protease inhibitors and then centrifuged at 55,000 $\times g$ for $3 \mathrm{~h}$ at $4^{\circ} \mathrm{C}$ in a swinging bucket rotor. Fractions $(1 \mathrm{ml})$ were collected from the top of the gradient and stored at $-80^{\circ} \mathrm{C}$ until use.

To identify proteins that cofractionated with mitochondria, gradient fractions were subjected to SDS-PAGE and Western blotting. After blocking in Tris-buffered saline/Tween 20 with $3 \%$ nonfat dry milk, blots were incubated with the following primary antibodies diluted in blocking solution: mouse anti-GFP (1:1000) (Clonetech), mouse anti-cytochrome $c$ (1:500) (BD Biosciences PharMingen, San Jose, CA), rabbit anti-Drosophila Khc (1:500) (Cytoskeleton), rat anti-p50/Dynamitin (1:200) (Duncan and Warrior, 2002), mouse anti-Drosophila Dhc64C (1:100) (Sharp et al., 2000), and rabbit antip150 Glued (1:2000) (Fan and Ready, 1997). Blots were washed in blocking solution and then incubated with the following secondary antibodies: horseradish peroxidase (HRP)-conjugated goat anti-rat $\operatorname{IgG}(1: 10,000)$, HRP-conjugated goat anti-rabbit IgG (1:10.000), and HRP-conjugated goat anti-mouse IgG $(1: 10,000)$ (Jackson ImmunoResearch Laboratories) diluted in blocking solution. Secondary antibody localizations were detected using chemiluminescence (Bio-Rad).

\section{RESULTS}

\section{Targeting GFP to Mitochondria in Drosophila \\ Motoneurons}

To make mitochondria visible in the axons of an intact nervous system, Drosophila were transformed with $P\left[w^{+m C}=U A S\right.$ mitoGFP.AP] (abbreviated as mitoGFP below), a fusion gene with a GAL4-responsive promoter and a mitochondrial import sequence fused to Green Fluorescent Protein(S65T) (Brand and Perrimon, 1993; Rizzuto et al., 1995). To drive mitoGFP expression in motoneurons, transgenic flies were crossed to mates carrying a tissue-specific Gal4 driver (Yeh et al., 1995), $P[$ GawB $] D 42$ (abbreviated as D42). Progeny with both the D42 driver and the mitoGFP responder showed fluorescence in neuronal tissues from late embryogenesis through adulthood. In dissected larvae, the pattern of GFP was consistent with expression in motoneurons and some other cells of the central nervous system (Figure 1A). At higher magnification, GFP in segmental nerves was seen concentrated in structures with the elongated appearance of mitochondria (Figure $1 \mathrm{~B})$.

To determine whether those structures were indeed mitochondria, the pattern of GFP was compared with that of MitoTracker Red. The abundance of mitochondria in larval glial cells and muscles made it difficult to distinguish individual MitoTracker-stained axonal mitochondria. To obtain clearer views, cells dissociated from homozygous D42 mitoGFP embryos were cultured, fixed, and stained. GFP fluorescence was restricted to a subset of cells, including many with long neuritic extensions. In those cells, the patterns of GFP and MitoTracker Red were indistinguishable (Supplemental Figure s1), confirming that the mitoGFP fusion protein was targeted to mitochondria.

To determine whether GFP-loaded mitochondria move, neurites of cultured neurons were imaged by time-lapse confocal microscopy (Supplemental Video 1). Some mitochondria seemed stationary, others displayed short saltatory movements with little net displacement, and some moved vigorously. The long-range movement consisted of resolute unidirectional runs separated by pauses. Most of those longrange mitochondria exhibited a directional bias. Runs in the 


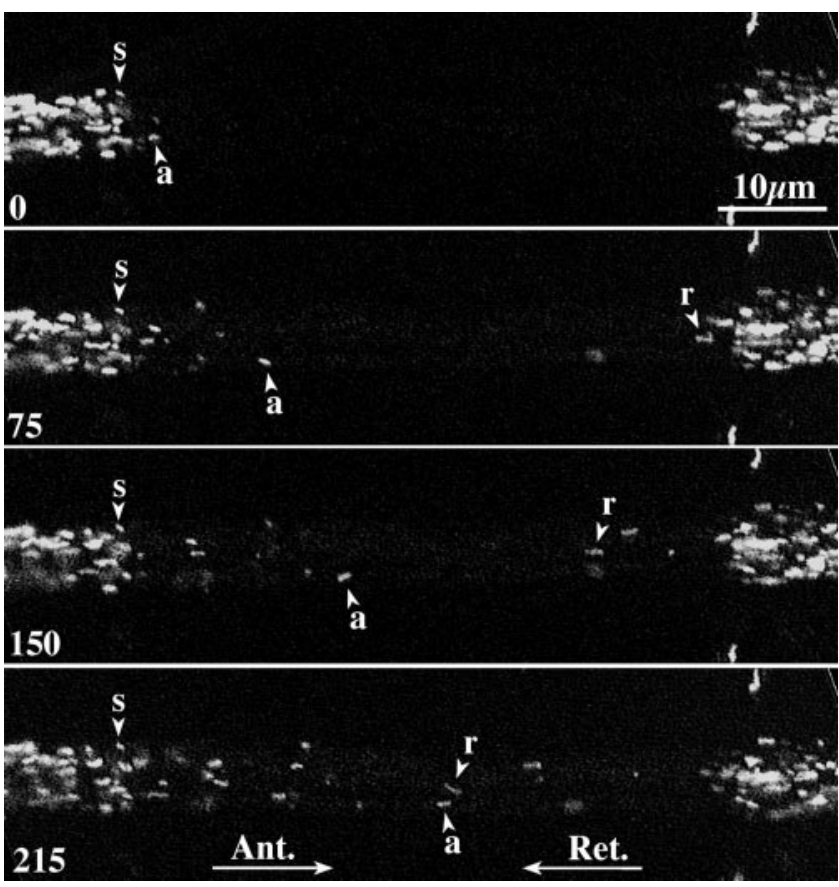

Figure 2. Transport of GFP-mitochondria in wild-type motor axons. A series of confocal images from a time-lapse movie of mitoGFP in motor axons of a segmental nerve in a dissected third instar (see Supplemental Video 2). The central $\sim 50-\mu \mathrm{m}$ region was photobleached and then imaged at the times indicated (seconds). Anterograde (Ant.) and retrograde (Ret.) directions are indicated by arrows. Stationary mitochondria (s) are evident outside the bleached zone. Anterograde mitochondria (a) entered the bleached zone from the left boundary and retrograde mitochondria (r) entered from the right.

primary direction of travel were more frequent than runs in the opposite direction. However, this directional bias was not absolute in cultured cells, i.e., conversions in primary direction were observed. The net velocity of anterograde mitochondria was $0.28 \pm 0.22 \mu \mathrm{m} / \mathrm{s}(\mathrm{n}=200$ tracking points, 11 mitochondria) with a peak of $0.75 \mu \mathrm{m} / \mathrm{s}$. The net velocity of retrograde mitochondria was $0.19 \pm 0.32 \mu \mathrm{m} / \mathrm{s}$ ( $\mathrm{n}=447$ tracking points, 11 mitochondria) with a peak of $1.51 \mu \mathrm{m} / \mathrm{s}$. Those values are consistent with transport by microtubule motors; however, the retrograde mean was lower than expected from prior studies of axonal mitochondria (Morris and Hollenbeck, 1993). This raised doubts about whether neurites of cultured embryonic neurons, which lack regular synaptic partners and glial support cells, have normal axonlike transport physiology.

\section{Mitochondrial Transport in True Motor Axons}

To investigate transport in an intact nervous system, GFPmitochondria were observed by time-lapse microscopy in the motor axons of larval segmental nerves, which are compound, containing 60-80 axons (Hurd and Saxton, 1996). To highlight mitochondria undergoing transport, a photobleaching approach was used. After locating a nerve, a $55-\mu \mathrm{m}$-long segment was photobleached, and then the movement of fluorescent mitochondria entering the bleached zone was recorded at $1 \mathrm{frame} / \mathrm{s}$ for $300 \mathrm{~s}$ (Figure 2 and Supplemental Video 2). Some mitochondria remained in view across the entire bleached zone. Others left or entered the confocal optical section at various points. Based on the number of different transport paths seen, an optical section of a nerve included five to 15 motor axons.

The directional imprinting for individual mitochondria in larval motor axons was strong, i.e., conversions in primary transport direction were never seen (104 mitochondria, 220 min of total tracking time). This allowed classification of organelles that entered the bleached zone from the cell body side (left side in all figures) as anterograde and those that entered from the terminal side as retrograde. The apparent absolute directional bias of individual mitochondria and the distinctive high velocity of their retrograde runs (see below) suggest that mitochondrial transport physiology in the axons of an intact nervous system is indeed different from that in the neurites of cultured cells. Assuming that transport in true axons better reflects normal mechanisms, further experimentation was focused on larval nerves.

\section{Anterograde Transport Bias in Motor Axons}

To estimate the proportions of stationary, anterograde, and retrograde mitochondria in axons, counts were made in four identical rectangles $60 \mathrm{~s}$ after photobleaching; two on either side of the anterior bleach zone boundary and two on either side of the posterior bleach zone boundary. By comparing organelle numbers in a bleached rectangle (moving mitochondria) and its neighboring unbleached rectangle (moving plus stationary mitochondria), it was determined that $57 \%$ of mitochondria were stationary, $29 \%$ moved anterograde, and $14 \%$ moved retrograde ( $\mathrm{n}=11$ nerves, 11 larvae). The twofold anterograde bias was reexamined by flux measurement; counts of the number of mitochondria entering the bleach zone per minute. Anterograde flux ranged from 2.0 to 6.8 mitochondria/min, and retrograde flux ranged from 1.6 to $3.8 / \mathrm{min}$. Comparison of means showed a $1.4-$ fold anterograde bias that was significant $(\mathrm{p}<0.048)$. The discrepancy between the two approaches reflects the fact that flux is determined by both the number of moving organelles and their net velocities. The number of retrograde mitochondria was approximately one-half that of anterograde, but retrograde net velocities were $\sim 50 \%$ higher.

Three possibilities for the anterograde flux bias were considered. First, mitochondria might fuse in the distal axon such that retrograde mitochondria were fewer but larger. One apparent fusion event was observed in cultured cell neurites, but neither fusion nor fission was seen in larval axons. Furthermore, mean organelle length for anterograde versus retrograde mitochondria in axons was not different (anterograde $=0.91 \pm 0.52 \mu \mathrm{m}, \mathrm{n}=52 ;$ retrograde $=1.08 \pm$ $0.78 \mu \mathrm{m}, \mathrm{n}=52 ; \mathrm{p}>0.05)$. Second, axonal mitochondria might be engulfed by autophagocytosis and degraded in lysosomes, before or during retrograde transport (Hollenbeck, 1993; Nixon and Cataldo, 1995; Tolkovsky et al., 2002). However, large autophagocytic and lysosomal organelles are rare in wild-type larval axons (Gho et al., 1992; Hurd and Saxton, 1996). A more likely explanation for the anterograde transport bias is physiological demand (Morris and Hollenbeck, 1993; Ruthel and Hollenbeck, 2003; Chada and Hollenbeck, 2004). During the 4-d period of larval development, axons and terminals must grow substantially to keep pace with a 25-fold increase in larval volume.

\section{Quantification of Mitochondrial Transport Parameters}

To address questions about transport mechanism in more detail, individual mitochondria were tracked over time and their behavior was parsed into runs and pauses (Figure 3 and Table 1). Runs in the primary direction of travel are termed "forward," and runs in the opposite direction are termed "reverse." Transport behavior was dominated by 


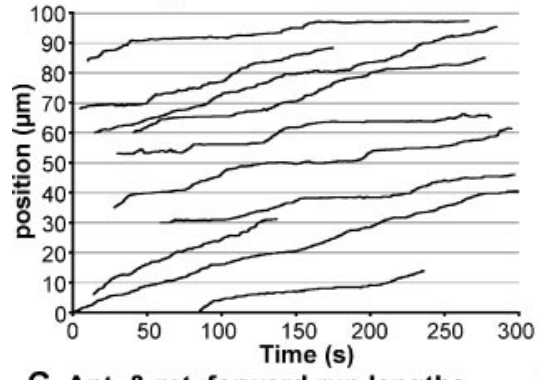

Figure 3. Comparison of transport parameters for anterograde and retrograde mitochondria. (A and $\mathrm{B}$ ) The positions of 10 randomly selected anterograde and retrograde mitochondria from nerves in five different wildtype larvae were plotted as a function of time. The starting position for each mitochondrion is arbitrary, selected to allow a clear view of each trace. Transport in both directions consisted of forward runs separated by pauses, short reverse runs, or both. Note the steeper but less regular slopes of the retrograde class of organelles. (C and D) The frequency distributions of forward run lengths (bins, 0.25 $\mu \mathrm{m}$ ) and velocities (bins, $0.05 \mu \mathrm{m} / \mathrm{s}$ ) for anterograde and retrograde mitochondria are shown. Note the clear bias of the anterograde class toward shorter, slower runs.
A. Anterograde mitochondria

\section{B. Retrograde mitochondria}

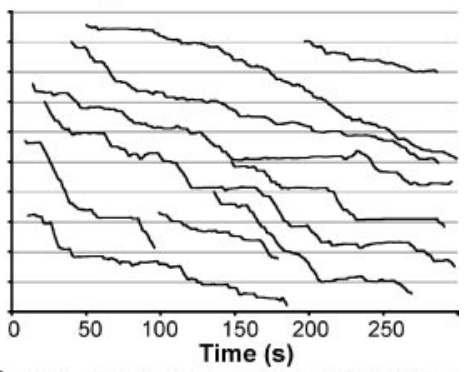

C. Ant. \& ret. forward run lengths

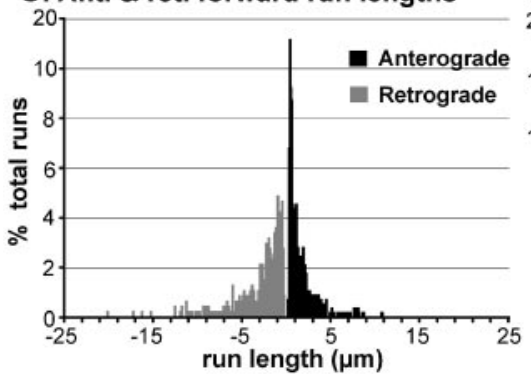

D. Ant. \& ret. forward run velocities

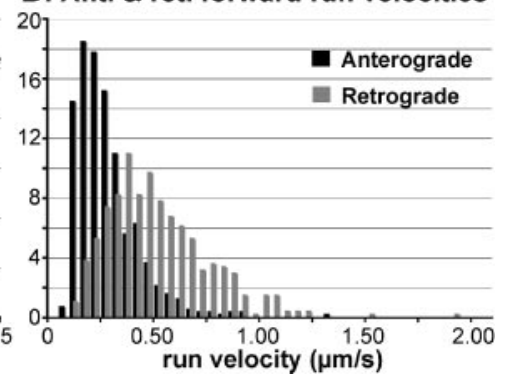

forward runs and pauses with relatively little time spent in reverse runs. Anterograde mitochondria spent $<1 \%$ of their time in reverse runs, whereas retrograde mitochondria spent $4 \%$ of their time in reverse runs, producing more irregular transport tracks (compare Figure 3, A and B). Mean forward run velocity for the anterograde class was $0.26 \pm 0.10 \mu \mathrm{m} / \mathrm{s}$. Retrograde class forward run velocity was almost twofold higher $(0.45 \pm 0.11 \mu \mathrm{m} / \mathrm{s})$. It was notable that although forward and reverse runs were often separated by pauses, rapid direction switching did occur, consistent with the idea that individual organelles can simultaneously bind both plus-end and minus-end motors (Hollenbeck, 1996; Welte et al., 1998; Kural et al., 2005).

The range of individual forward run velocities within a class was broad (anterograde, 0.1-1.79 $\mu \mathrm{m} / \mathrm{s}$; retrograde, $0.1-2.86 \mu \mathrm{m} / \mathrm{s}$ ), suggesting that multiple motor types with different intrinsic velocities might contribute to the transport mechanisms. Candidate retrograde motors in Drosophila include cytoplasmic dynein and Ncd, a kinesin-14. The most likely anterograde motors include members of the kinesin-1, -2 , and -3 families. Frequency distributions for forward run velocities and lengths showed distinct profiles for anterograde versus retrograde mitochondria, consistent with a polarized microtubule array and class-specific transport motors $(\mathrm{p}<$ 0.01). However, clear indications of multiple types of motors within a class, such as multiple run length or run velocity peaks, were not seen (Figure 3, C and D).

\section{Cytoplasmic Dynein Is Critical for Retrograde Transport}

To identify microtubule motors that move axonal mitochondria, the effects of genetic mutations were studied. To test dynein, a hypomorphic allele of Dynein heavy chain $64 \mathrm{C}$ $\left(D h c 64 C^{6-10}\right)$ was used in combination with an amorphic allele $\left(D h c 64 C^{4-19}\right)$. This hypomorphic genotype avoids the pleiotropic early physiological defects caused by Dhc64C amorphic/null genotypes (Gepner et al., 1996), allowing development of classic neuronal phenotypes, including organelle-filled axonal swellings, paralytic tail flipping, and axon terminal atrophy (Martin et al., 1999a). By observing and tracking mitochondria in regions of Dhc64C mutant nerves that lacked obvious swellings, it was evident that retrograde flux was reduced more than sixfold (Figures 4A and 5, Table 1, and Supplemental Video 3). The few mitochondria that did move retrograde had 43 and 30\% decreases, respectively, in forward run length and duration (Figure 4B and Table 1). They also showed a substantial shift from time spent in runs to time spent in pauses (Figure 6 and Table 1). These results, derived from a hypomorphic genotype that avoided the pleiotropic physiological defects caused by null genotypes, argue that cytoplasmic dynein is the primary motor for long-distance transport of axonal mitochondria toward the neuronal cell body.

\section{Axonal Swellings Are Not Static Blockades}

In previous studies of the effects of kinesin-1, dynein, and dynactin mutations, we proposed that stationary organelles stalled by weak motor function caused "traffic jams" that grew into axonal swellings through simple blockage of both anterograde and retrograde organelle passage (Hurd and Saxton, 1996; Martin et al., 1999a). Consistent with this, Dhc64C mutations, like Khc mutations, cause substantial accumulations of both synaptic vesicle precursors, thought to reflect stalled anterograde transport, and large prelysosomal bodies, thought to reflect stalled retrograde transport (Hurd and Saxton, 1996; Martin et al., 1999a). Several observations from our time-lapse imaging of GFP-mitochondria refute that traffic jam hypothesis. First, although in wild-type axons $\sim 50 \%$ of mitochondria were stationary (Table 1 and Supplemental Video 2), swellings were rare (Hurd and Saxton, 1996; Martin et al., 1999a). Second, mitochondria in obvious swellings were usually quite mobile and could exit as well as enter (Supplemental Video 4). Third, despite the presence of numerous swellings in Dhc64C mutant nerves, there were no significant changes in anterograde flux or anterograde forward run parameters (Figures $4 \mathrm{~A}$ and 5 and Table 1). Thus, steric hindrance from stationary organelles and swellings does not have a major influence on the transport of mitochondria in regions of axons that lack swellings.

An additional argument that the effects of dynein mutations on transport are direct comes from tracking of mitochondria in axons of $D h c 64 C^{4-19} /+$ larvae (Supplemental 
Table 1. Transport parameters for mitochondria in segmental nerve motor axons

\begin{tabular}{|c|c|c|c|c|c|c|}
\hline \multirow{2}{*}{$\begin{array}{l}\text { Genotype } \\
\text { Organelle class }\end{array}$} & \multicolumn{2}{|c|}{ Wild-type } & \multicolumn{2}{|c|}{$D h c 64 C^{6-10} / D h c 64 C^{4-19}$} & \multicolumn{2}{|c|}{$K h c^{6} / K h c^{27}$} \\
\hline & Anterograde & Retrograde & Anterograde & Retrograde & Anterograde & Retrograde \\
\hline No. larvae (no. Mito) & $5(52)$ & $5(52)$ & $5(92)$ & $4(11)$ & $4(24)$ & $2(5)$ \\
\hline Total time (min) & 120.5 & 102.9 & 176.15 & 19.15 & 51.22 & 5.75 \\
\hline Flux & $4.02 \pm 1.83$ & $2.75 \pm 0.80$ & $4.64 \pm 2.0$ & $0.44 \pm 0.36^{*}$ & $1.00 \pm 0.76^{*}$ & $0.28 \pm 0.40^{*}$ \\
\hline \multicolumn{7}{|l|}{ Anterograde runs } \\
\hline$\%$ Tot. time & $52.30 \pm 19.64$ & $3.57 \pm 4.78$ & $54.79 \pm 14.67$ & $1.76 \pm 2.81$ & $50.31 \pm 19.78$ & $4.03 \pm 3.99$ \\
\hline Velocity $(\mu \mathrm{m} / \mathrm{s})$ & $0.26 \pm 0.10$ & $0.20 \pm 0.07$ & $0.22 \pm 0.04^{*}$ & $0.16 \pm 0.09$ & $0.24 \pm 0.06^{*}$ & $0.19 \pm 0.04$ \\
\hline Duration (s/run) & $6.41 \pm 2.43$ & $2.59 \pm 0.82$ & $6.65 \pm 2.19$ & $2.29 \pm 0.64$ & $6.47 \pm 3.81$ & $2.53 \pm 0.50$ \\
\hline Length ( $\mu \mathrm{m} /$ run $)$ & $1.82 \pm 1.19$ & $0.51 \pm 0.21$ & $1.54 \pm 0.66$ & $0.36 \pm 0.21$ & $1.72 \pm 1.32$ & $0.46 \pm 0.04$ \\
\hline \multicolumn{7}{|l|}{ Retrograde runs } \\
\hline$\%$ total time & $0.87 \pm 2.04$ & $51.85 \pm 17.43$ & $0.63 \pm 2.17$ & $34.92 \pm 25.18^{*}$ & $1.97 \pm 3.19$ & $50.51 \pm 26.04$ \\
\hline Velocity $(\mu \mathrm{m} / \mathrm{s})$ & $0.23 \pm 0.11$ & $0.45 \pm 0.11$ & $0.21 \pm 0.05$ & $0.36 \pm 0.16$ & $0.24 \pm 0.07$ & $0.35 \pm 0.12$ \\
\hline Duration (s/run) & $2.64 \pm 1.04$ & $6.66 \pm 2.84$ & $2.33 \pm 0.44$ & $4.67 \pm 1.06^{*}$ & $2.90 \pm 1.08$ & $5.83 \pm 2.75$ \\
\hline Length $(\mu \mathrm{m} / \mathrm{run})$ & $0.64 \pm 0.47$ & $3.14 \pm 1.61$ & $0.48 \pm 0.18$ & $1.78 \pm 0.93^{*}$ & $0.71 \pm 0.30$ & $2.34 \pm 1.67$ \\
\hline \multicolumn{7}{|l|}{ Pauses } \\
\hline$\%$ total time & $46.82 \pm 19.35$ & $44.58 \pm 16.12$ & $44.59 \pm 14.55$ & $63.31 \pm 24.70^{*}$ & $47.72 \pm 19.39$ & $45.46 \pm 22.31$ \\
\hline Duration (s) & $6.15 \pm 3.90$ & $5.70 \pm 3.26$ & $5.71 \pm 3.88$ & $14.78 \pm 17.19$ & $5.93 \pm 3.09$ & $4.86 \pm 2.29$ \\
\hline Genotype & \multicolumn{2}{|c|}{$G l^{1} /+$} & \multicolumn{2}{|c|}{ Glued RNAi } & \multicolumn{2}{|c|}{$K h c^{17} / K h c^{27}$} \\
\hline Organelle class & Anterograde & Retrograde & Anterograde & Retrograde & Anterograde & Retrograde \\
\hline No. larvae (no. Mito) & $5(94)$ & $5(64)$ & $5(85)$ & $5(67)$ & $2(8)$ & $2(6)$ \\
\hline Total time (min) & 153.22 & 95.87 & 176.25 & 105.98 & 12.03 & 12.37 \\
\hline Flux & $4.72 \pm 1.45$ & $2.80 \pm 1.59$ & $4.68 \pm 2.41$ & $2.29 \pm 1.60$ & $0.40 \pm 0.40^{*}$ & $0.40 \pm 0.53^{*}$ \\
\hline \multicolumn{7}{|l|}{ Anterograde runs } \\
\hline$\%$ total time & $62.66 \pm 19.8^{*}$ & $2.27 \pm 3.61$ & $56.95 \pm 20.39$ & $3.49 \pm 4.49$ & $64.47 \pm 19.04$ & $5.77 \pm 4.03$ \\
\hline Velocity $(\mu \mathrm{m} / \mathrm{s})$ & $0.31 \pm 0.11^{*}$ & $0.25 \pm 0.12 *$ & $0.33 \pm 0.10^{*}$ & $0.28 \pm 0.11^{*}$ & $0.26 \pm 0.04$ & $0.26 \pm 0.09$ \\
\hline Duration (s/run) & $6.91 \pm 3.90$ & $2.37 \pm 0.41$ & $6.99 \pm 2.70$ & $2.42 \pm 0.50$ & $7.66 \pm 2.94$ & $2.68 \pm 0.54$ \\
\hline Length $(\mu \mathrm{m} /$ run $)$ & $2.30 \pm 1.61$ & $0.58 \pm 0.27$ & $2.52 \pm 1.30^{*}$ & $0.67 \pm 0.31^{*}$ & $2.10 \pm 0.86$ & $0.66 \pm 0.26$ \\
\hline \multicolumn{7}{|l|}{ Retrograde runs } \\
\hline$\%$ total time & $1.00 \pm 2.04$ & $55.93 \pm 24.06$ & $0.80 \pm 1.72$ & $52.85 \pm 21.33$ & $0.40 \pm 1.12$ & $53.87 \pm 30.07$ \\
\hline Velocity $(\mu \mathrm{m} / \mathrm{s})$ & $0.22 \pm 0.07$ & $0.54 \pm 0.18^{*}$ & $0.27 \pm 0.08$ & $0.62 \pm 0.19^{*}$ & 0.31 & $0.49 \pm 0.13$ \\
\hline Duration (s/run) & $2.36 \pm 0.90$ & $6.65 \pm 3.10$ & $2.37 \pm 0.61$ & $6.64 \pm 3.15$ & 2.50 & $9.30 \pm 10.20$ \\
\hline Length ( $\mu \mathrm{m} / \mathrm{run})$ & $0.53 \pm 0.25$ & $4.02 \pm 2.76$ & $0.64 \pm 0.25$ & $4.51 \pm 2.81^{*}$ & 0.77 & $5.24 \pm 5.98$ \\
\hline \multicolumn{7}{|l|}{ Pauses } \\
\hline$\%$ total time & $36.34 \pm 19.39^{*}$ & $41.80 \pm 22.70$ & $42.26 \pm 19.77$ & $43.66 \pm 20.09$ & $35.13 \pm 18.07$ & $40.36 \pm 28.9$ \\
\hline Duration (s) & $4.25 \pm 3.17^{*}$ & $6.02 \pm 6.58$ & $5.89 \pm 4.99$ & $6.14 \pm 4.72$ & $4.50 \pm 2.63$ & $6.12 \pm 4.32$ \\
\hline
\end{tabular}

The center of each mitochondrion that entered a bleached zone by fast transport was marked in every frame (300 at $1 / \mathrm{sec})$ of five movies from five separate larvae for each genotype. Flux, run, and pause values reflect means \pm SD. Some mutant larva sample sizes were less than five, because in some tests, no mitochondria of a particular directional class entered the bleached zone. Mutant parameters that were judged significantly different from wild type by linear contrast $(p<0.05)$ are bold and followed by an asterisk $\left(^{*}\right)$. Sample sizes for Khc ${ }^{17} / K h c^{27}$ were small and variances were large; consequently, other than for flux values, $K h c^{17} / K h c^{27}$ statistical comparisons were not robust.

Results of particular note: 1) Dhc mutations inhibited retrograde flux and retrograde runs, suggesting that dynein is the primary retrograde motor; 2) Khc mutations inhibited anterograde and retrograde flux, suggesting that kinesin-1 is the primary anterograde motor and that it is required for dynein activity in axons; 3) Dhc mutations did not enhance anterograde runs and Khc mutations did not enhance retrograde runs, suggesting that dynein and kinesin-1 generated forces alternate rather than opposing one another; and 4) $\mathrm{Gl}^{1}$ mutations and Glued RNAi enhanced anterograde and retrograde runs, suggesting that axonal P150 Glued retards runs by kinesin-1 and dynein.

Table 1), which showed few or no swellings. The amorphic allele, even when heterozygous, caused significant reductions in retrograde run lengths and durations. This confirms the results from Dhc64C $4-19 / D h c 64 C^{6-10}$ without the complications of axonal swellings and pleiotropic physiological defects that can arise from severe loss of function genotypes. It also suggests that the tracking and statistical approaches used were sufficiently sensitive to detect subtle changes in transport mechanisms. In summary, although the possibility of contributions from minus-end-directed kinesins and cytoplasmic myosins remains, our results suggest that cytoplasmic dynein is the primary long-distance retrograde motor for axonal mitochondria.

\section{Kinesin-1 Is Critical for Both Anterograde and Retrograde Transport}

To assess the influence of the sole Drosophila kinesin-1 on transport, Khc mutations were tested. Two different hypomorphic $K h c$ alleles $\left(K h c^{6}\right.$ or $\left.K h c^{17}\right)$ were used in combination with a null allele $\left(K h c^{27}\right) . K h c^{6}$ alters coil 2 of the Khc stalk (Rose and Saxton, unpublished data), a region thought to be important for binding kinesin light chain and for linkage to cargoes. $K h c^{17}$ alters loop 11 of the motor domain, inhibiting catalytic turnover rate and causing a 1.6-fold reduction of motor velocity in vitro (Brendza et al., 1999). In $K h c^{6} /$ $K h c^{27}$ and $K h c^{17} / K h c^{27}$ nerves, it was striking that few mitochondria moved in either direction (Supplemental Video 5). 
A. Dhc mutant

Figure 4. Dynein mutations disrupt retrograde transport of mitochondria. (A) A series of confocal images from a time-lapse movie of mitochondria in motor axons of a larva carrying Dhc64C $C^{6-10} / D_{c} 64 C^{4-19}$ (Supplemental Video 3). Note that the number of retrograde mitochondria $(r)$ that entered the photobleached zone was substantially reduced relative to anterograde mitochondria (a). A stationary mitochondrion (s) near the anterior bleach boundary is marked for reference. (B) Frequency distributions are plotted for two forward run parameters of the five retrograde mitochondria that entered bleached zones in 10 different Dhc64C mutant larvae (gray bars). Black line-tracings show analogous frequencies for wild-type mitochondria. Note that despite the small sample size from mutants, a significant shift to shorter retrograde runs is evident $(\mathrm{p}<0.05$; see Table 1$)$.

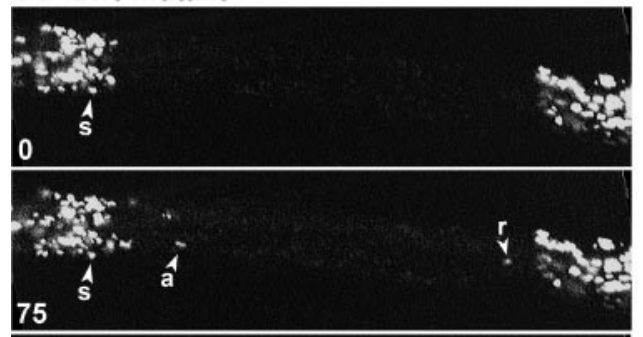

\section{B. Retrograde class}
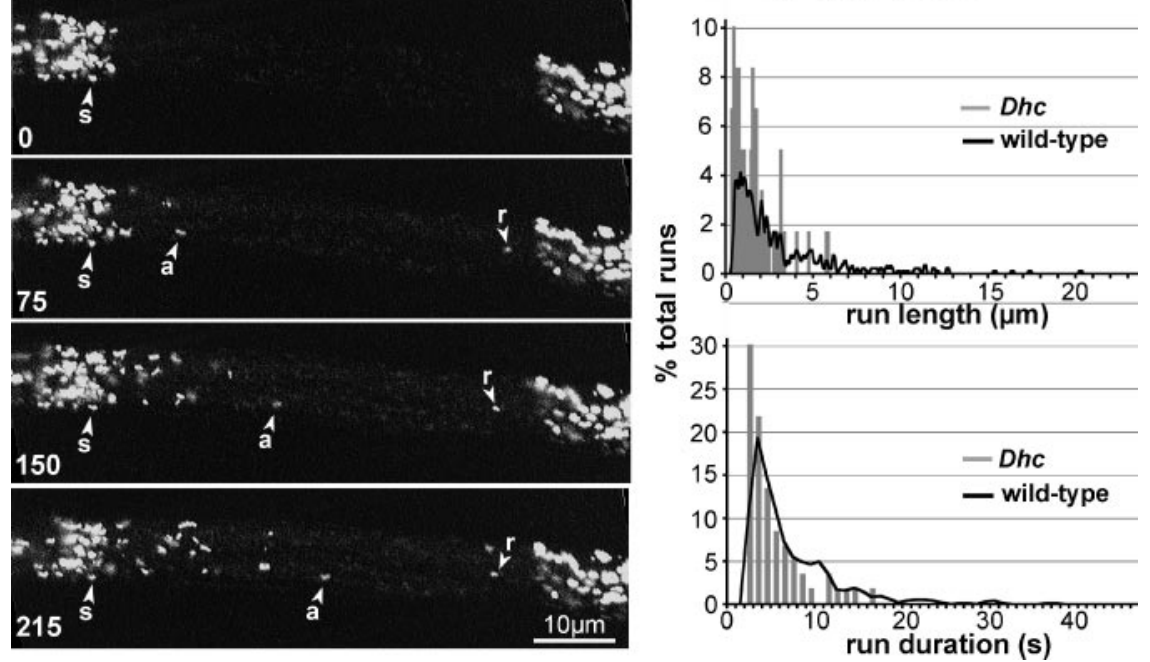

Anterograde and retrograde flux reductions ranged from 70 to 90\% (Figure 5 and Table 1). With the exception of the anterograde class in $K h c^{6} / K h c^{27}$ nerves, sample sizes were small (i.e., $\leq 2$ larvae, 8 mitochondria, and 8 forward runs total per class). Hence, strong interpretations of most Khc mutant transport parameter means are not warranted. For the $K h c^{6} / K h c^{27}$ anterograde mitochondria, there was a significant reduction in anterograde run velocity, but it was not substantial (8\%). Perhaps the $\mathrm{Khc}^{6}$ protein is impaired in initial cargo attachment, but once bound, can drive normal anterograde motion. It is also possible that residual wild-type maternal Khc contributed to transport (Saxton et al., 1991).

The dramatic reduction in anterograde flux observed in the Khc mutant animals is consistent with the idea that plus-end-

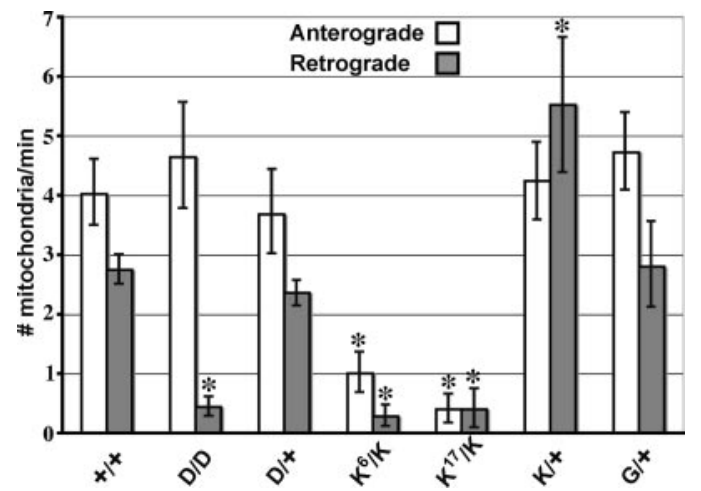

Figure 5. Effects of dynein, kinesn-1 and dynactin mutations on anterograde and retrograde flux of mitochondria. The mean numbers $( \pm \mathrm{SD})$ of anterograde or retrograde mitochondria entering bleach zones per minute are shown for the following genotypes:

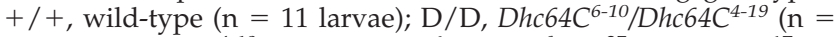
5); $\mathrm{D} /+, D_{c} 64 C^{4-19} /+(\mathrm{n}=5) ; \mathrm{K}^{6} / \mathrm{K}, \mathrm{Khc}^{6} / \mathrm{Khc}^{27}(\mathrm{n}=5) ; \mathrm{K}^{17} / \mathrm{K}$, $K h c^{17} / K^{2} c^{27}(\mathrm{n}=3) ; \mathrm{K} /+, K h c 27 /+(\mathrm{n}=10) ;$ and $\mathrm{G} /+, G l^{1} /+(\mathrm{n}=5)$. Note that flux is a compound parameter determined by net organelle velocities, the abundance of organelles in axons, and by the fraction of axonal organelles moving in a given direction. Numerical values are shown in Table 1 and Supplemental Table s1. To determine whether flux values were significantly different from wild type, one-way descriptive statistic linear contrast tests using aggregated larval data were used. Means showing a genotype by genotype contrast with $p<0.05$ were accepted as significantly different from wild type $\left.{ }^{*}\right)$.
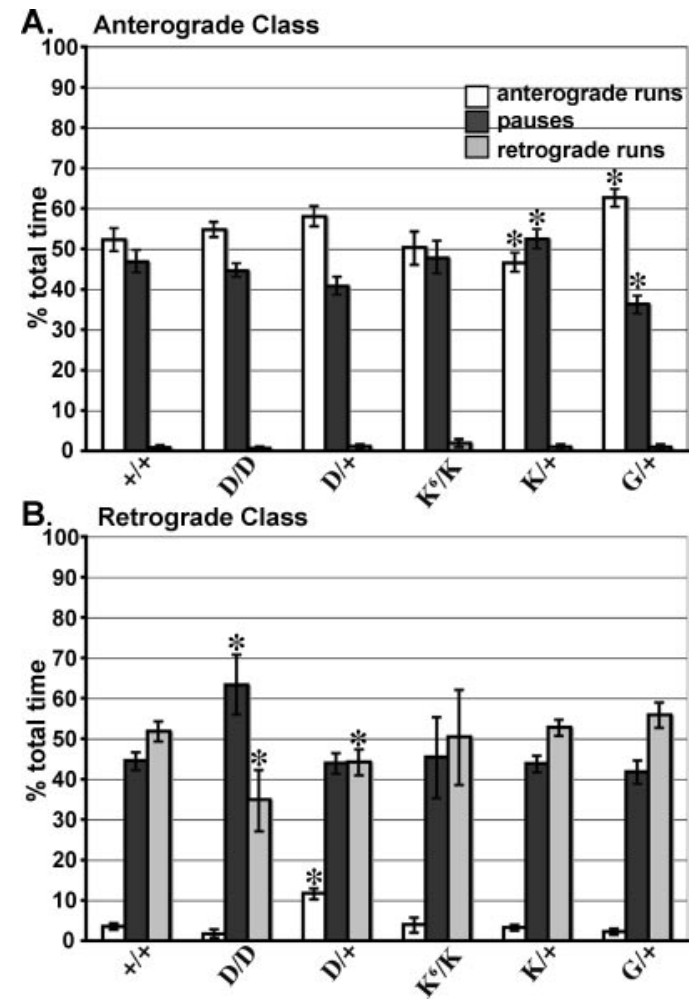

Figure 6. Effects of dynein, kinesn-1, and dynactin mutations on anterograde and retrograde class duty cycles. The percentage of time spent in anterograde runs, pauses, and retrograde runs for anterograde and retrograde class mitochondria are shown for the following genotypes: $+/+$, wild-type (for both $\mathrm{A}$ and $\mathrm{B}, \mathrm{n}=52$ mitochondria in 5 larvae); $\mathrm{D} / \mathrm{D}, \mathrm{Dhc} 64 \mathrm{C}^{6-10} / \mathrm{Dhc} 64 \mathrm{C}^{4-19}$ (for $\mathrm{A}, \mathrm{n}=$ 92 and 5; for $\mathrm{B}, \mathrm{n}=11$ and 5); $\mathrm{D} /+, D h c 64 C^{4-19} /+$ (for $\mathrm{A}, \mathrm{n}=44$ and 5 ; for $\mathrm{B}, \mathrm{n}=43$ and 5); $\mathrm{K}^{6} / \mathrm{K}, K h c^{6} / K h c^{27}$ (for $\mathrm{A}, \mathrm{n}=24$ and 4 ; for $\mathrm{B}$, $\mathrm{n}=5$ and 4$) ; \mathrm{K} /+, K h c 27 /+($ for $\mathrm{A}, \mathrm{n}=85$ and $5 ;$ for $\mathrm{B}, \mathrm{n}=118$ and 5); and $\mathrm{G} /+$ ) $G l^{1} /+$ (for $\mathrm{A}, \mathrm{n}=94$ and 5; for $\mathrm{B}, \mathrm{n}=64$ and 5). Bars show SEs. Values can be found in Table 1 and Supplemental Table s1. Means showing a genotype by genotype contrast significance of $\mathrm{p} \leq 0.05$ compared with wild type are noted by an asterisk. 
A
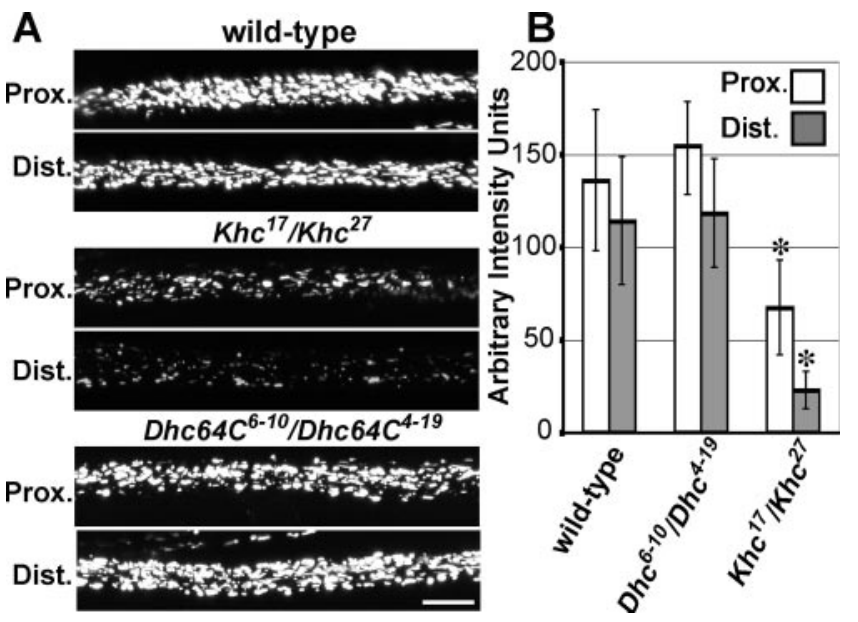

Figure 7. Mutations in kinesin and dynein affect the distribution of mitochondria in segmental nerves. (A) Single confocal optical sections of GFP-mitochondria in motor axons of segmental nerve A8 in live preparations. Imaging parameters were identical for all preparations. Images from the anterior segment A2, proximal to motoneuron cell bodies (Prox.), and from the posterior segment A7, distal to the cell bodies (Dist.), are shown. (B) To quantify the effects of Khc and Dhc64C mutations on the number of mitochondria in proximal and distal regions, fluorescence intensity per unit area was measured in proximal and distal regions of A8 nerves from three larvae for each genotype ( $\mathrm{n}=20$ proximal and 20 distal measurements/ larva, $34.5 \mu \mathrm{m}^{2} /$ measurement). Bars show mean intensity values $( \pm S D)$. Means significantly different from wild type $(\mathrm{p}<0.05)$ are noted with an asterisk. Bar (A), $10 \mu \mathrm{m}$.

directed kinesin- 1 is a major motor for axonal mitochondria. However, there was also a dramatic reduction in retrograde flux, most of which reflects minus-end-directed transport by cytoplasmic dynein. Because we saw no signs of mitochondrial fission in axons and because there is evidence that biogenesis occurs mainly near the nucleus (Davis and Clayton, 1996), it is reasonable to think that mitochondria must be carried into axons by kinesin- 1 before they can engage in dynein-driven retrograde transport. If so, then $K h c$ mutants should have fewer mitochondria in their axons. To address that possibility, the relative fluorescence intensities of mito-GFP in segmental nerve $\mathrm{A} 8$ were determined for wild-type and $K h c^{17} / K_{h} c^{27}$ larvae (Figure 7 and Supplemental Table s2). Compared with wild-type, mean fluorescence intensity, hence the number of mitochondria, in the mutant nerves was decreased $50.5 \%$ in segments A2-3. This explains a portion of the $90 \%$ reduction of retrograde flux measured in $K h c^{17} / K h c^{27}$ nerves (Figure 5) but not all of it. The remainder may reflect a more direct functional dependence of cytoplasmic dynein on kinesin-1 (Martin et al., 1999a; Brendza et al., 2002; Ligon et al., 2004).

To test the effects of a milder reduction in Khc activity, mitochondrial movements were tracked in $K h c^{27} /+$ nerves. Mitochondria were abundant, and axonal swellings were not evident. There was a small shift in the anterograde duty cycle from forward runs to pauses (Figure 6A and Supplemental Table s1) and a $16 \%$ decrease in anterograde run duration, suggesting that lowered Khc dosage reduced anterograde persistence of mitochondria. There were no significant changes in retrograde class velocities or duty cycles. There was, however, an increase in retrograde flux (Figure 5 and Supplemental Table s1), suggesting a regulatory shift that elevated the number of mitochondria moving retrograde at the expense of the stationary class. Such an imbalance favoring retrograde flux could not be a sustained state during larval development, because it would deplete mitochondria from the axons. No obvious depletion was observed. Thus, it was likely a temporary state, perhaps induced by developmental or other physiological cues (e.g., Chada and Hollenbeck, 2003) altered by the Khc genotype or by unknown genetic variables. This highlights the importance of future investigations of developmental profiles for mitochondrial flux, the regulatory systems that control shifts between classes, and whether those systems are influenced directly by motors.

\section{P150 Glued Influences Anterograde and Retrograde Transport Parameters}

The dynactin complex is well known for a physical association with cytoplasmic dynein and for positive functional effects on dynein-mediated processes (reviewed by Schroer, 2004). Consistent with this, genetic tests of Glued ( $G l)$, which encodes the Drosophila P150Glued dynactin component, indicate a synergistic functional relationship with Dhc64C in fast axonal transport. However, $\mathrm{Gl}$ has an even stronger synergistic axonal transport relationship with Khc (Martin et al., 1999a). Biophysical studies of lipid droplet transport in Drosophila embryos with the dominant-negative $G l^{1}$ allele and with hypomorphic Dhc64C alleles suggest that P150Glued acts as a motor coordination factor on lipid droplets, preventing simultaneous opposing force production by dynein and an unknown anterograde motor (reviewed by Gross, 2003).

To assess the contributions of P150 Glued to mitochondrial transport, we tested the effects of the $G l^{1}$ allele. As described previously, $G l^{1} /+$ larvae do not show tail flipping and axonal swelling phenotypes (Martin et al., 1999a). Our timelapse tracking analysis of mitochondria in motor axons showed significant increases in anterograde and retrograde forward run parameters, rather than the decreases one would expect if P150Glued acted as either a simple dynein activation factor or as a kinesin-1/dynein coordination factor (Table 1). For anterograde mitochondria, the duty cycle showed a significant shift from pauses to forward runs and forward (anterograde) run velocity was increased. For retrograde mitochondria, duty cycles were not detectably affected, but there was a significant increase in forward (retrograde) run velocity.

The run parameter increases caused by $\mathrm{Gl}^{1} /+$ may not reflect a simple inhibition of P150 Glued in motoneurons. First, although the $G l^{1}$ allele produces a truncated protein that acts as a dominant-negative inhibitor of P150 Glued in eye development (McGrail et al., 1995), it might have an aberrant gain of function effect in axonal transport. Second, the increases could reflect an indirect $G l^{1}$ effect that alters axonal transport regulatory signals to axons from glial or other cells. Third, the increases could be due to unknown genetic background effects. To address those possibilities, we used a tissue-specific RNAi approach. Flies were generated that carried a UAS-Glued-snapback RNAi transgene along with UAS-mitoGFP and the D42-Gal4 motoneuron driver. Like $G l^{1} /+$, GluedRNAi flies were viable and showed little evidence of paralysis or axonal swellings. However, tracking of mitochondria in GluedRNAi larval axons showed significant increases in anterograde and retrograde forward run velocities and lengths (Table 1). Although less severe, the RNAi effects suggest that the $G l^{1}$ results do represent a dominant negative phenotype in motoneurons. The two approaches agree, suggesting that normal P150Glued in motor axons reduces run velocity and persistence for both dynein- and kinesin-1-driven transport of mitochondria. 


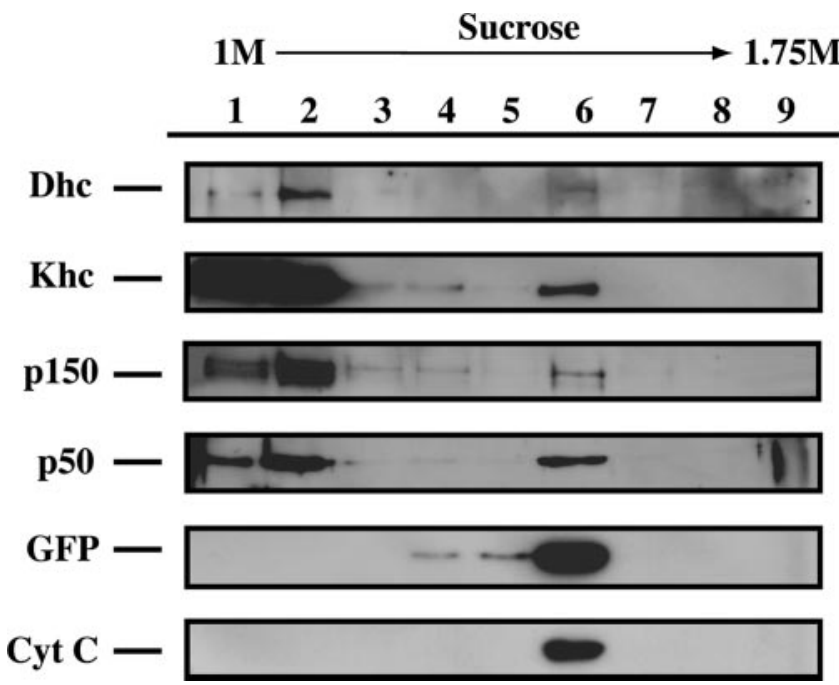

Figure 8. Cofractionation of dynein, kinesin-1 and dynactin components with neuronal mitochondria. Cytoplasm containing mitochondria from adult flies homozygous for D42 and mitoGFP was initially fractionated by differential sedimentation (Supplemental Figure s3) and then fractionated further by equilibrium density gradient centrifugation. Western blots of gradient fractions are shown stained with antibodies specific for cytoplasmic dynein (Dhc), kinesin-1 (Khc), and the dynactin components P150 Glued (p150) and p50/dynamitin (p50). Fractions containing mitochondria were identified by anti-GFP and anti-cytochrome $c(\mathrm{CytC})$ staining.

\section{Physical Association of Kinesin-1 and Cytoplasmic Dynein with Mitochondria}

If kinesin-1, dynein, and P150Glued all contribute to normal mitochondrial transport behavior in axons, they should all associate with mitochondria. To test this, adult flies with D42-Gal4-driven neuronal expression of mitoGFP were homogenized and fractionated by differential sedimentation. A fraction enriched for neuronal mitochondria, as evidenced by the presence of GFP and endogenous cytochrome $c$ (Supplemental Figure s2), was fractionation further by equilibrium sucrose density gradient centrifugation. Western blots of fractions showed the presence of some Khc, and Dhc64C spread throughout the gradient, suggesting their association with a variety of cytoplasmic components. However, Khc and Dhc64C as well as p150Glued and p50/Dynamitin were most abundant in the fraction with the highest levels of mitochondrial markers (Figure 8). This and coimmunolocalization tests in nerves (Supplemental Figure s3) support the view that kinesin 1, cytoplasmic dynein, and the dynactin complex do physically associate with neuronal mitochondria.

\section{DISCUSSION}

\section{Motors for Axonal Transport of Mitochondria}

To address questions about mechanisms of filament-based organelle transport, we developed a system to image and track mitochondria in an intact Drosophila nervous system. Our results indicate that although they can rapidly switch between anterograde runs, pauses, and retrograde runs, individual axonal mitochondria are strongly programmed for one of three states: net anterograde motion, stationary, or net retrograde motion. No direct switching between anterograde and retrograde classes was observed. Some conversions to the stationary class may have been seen, but the duration of our movies (300 s) made it difficult to distinguish such conversions from long pauses. Mutant analyses suggest that the primary motors for mitochondrial movement in larval motor axons are kinesin-1 (anterograde) and cytoplasmic dynein (retrograde), and interestingly that kinesin-1 is critical for retrograde transport by dynein. During transport, there was no evidence that force production by the two opposing motors was competitive, suggesting a mechanism for alternate coordination. Tests of the possible coordination factor P150Glued suggest that it indeed influences both motors on axonal mitochondria, but we saw no evidence that its function is critical for the motor coordination mechanism.

Cytoplasmic dynein, the most ubiquitous minus-end microtubule motor, has been regarded as a likely retrograde axonal transport motor for many years, but tests of that possibility have been largely indirect. For example, either EHNA or vanadate, which both inhibit dynein and other enzymes, inhibits axonal transport (Ekstrom and Kanje, 1984; Wang et al., 1995; Lalli et al., 2003). More specific dynein inhibition by genetic mutation causes changes in axonal organelle distributions consistent with transport defects (Bowman et al., 1999; Martin et al., 1999a; Koushika et al., 2004). The live analysis here shows that dynein mutations inhibit specific retrograde run parameters and flux but that they leave anterograde transport largely unchanged. This relatively direct evidence, combined with the lack of evidence for an alternative fast minus-end motor, provides a strong case for cytoplasmic dynein as the primary motor for long-distance retrograde mitochondrial transport in axons.

Multiple plus-end microtubule motors could contribute to mitochondrial transport, but the most likely candidates in axons are members of the kinesin- 1 and kinesin- 3 subfamilies (Hirokawa and Takemura, 2005). $\mathrm{Kif1B} \alpha$, a mouse kinesin-3, can bind mitochondria and move them on microtubules in vitro (Nangaku et al., 1994), and genetic tests in Neurospora show that normal mitochondrial behavior requires a kinesin-3 (Fuchs and Westermann, 2005). Kinesin-1 motors associate with mitochondria (Leopold et al., 1992; Khodjakov et al., 1998), and a mouse kinesin-1 (Kif5) is needed to prevent mitochondria from clustering near the cell centers of extraembryonic cells, the presumed location of microtubule minus-ends (Tanaka et al., 1998; Kanai et al., 2000). More directly relevant to our study, two Drosophila proteins thought to mediate linkage of kinesin-1 to mitochondria (Milton and Miro) are required for the accumulation of mitochondria in larval axons (Stowers et al., 2002; Guo et al., 2005). Our results with two different hypomorphic Khc mutant genotypes indicate that inhibition of kinesin-1 severely limits anterograde mitochondrial transport. The residual transport might be due to a kinesin-3, to traces of maternally provided wild-type kinesin, or to partial function of the mutant Khc proteins. Regardless, our findings combined with the Miro and Milton findings provide a strong case for kinesin-1 as the primary long-range anterograde mitochondrial transport motor in Drosophila axons.

\section{The Influence of Kinesin-1 on Dynein-driven Retrograde Transport}

The negative impact of the hypomorphic Khc mutant genotypes on retrograde mitochondrial transport raises questions about the relationship of anterograde and retrograde motors. We offer three nonexclusive explanations. First, if biogenesis of mitochondria is restricted to the vicinity of the nucleus in neurons (Davis and Clayton, 1996), mitochondria must be transported from the cell body into the axon by kinesin- 1 before dynein can transport them back. Second, because most protein synthesis is thought to occur in the somato-dendritic region and most axonal microtubules have plus 
ends distal, anterograde transport by kinesin- 1 may be required for maintaining normal levels of dynein in the distal axon (Pfister, 1999; Susalka et al., 2000). A third possibility is that kinesin-1 acts as a biochemical or biophysical activator of dynein in axons (Martin et al., 1999a). In support of a direct interaction, it has been reported that dynein intermediate chains can bind kinesin-1 light chains (Ligon et al., 2004). Such a physical/functional relationship could explain the striking observation that Khc-specific antibodies, when injected into cultured neurons or perfused into extruded axoplasm, cause a rapid inhibition of organelle transport in both directions (Brady et al., 1990; Theiss et al., 2005).

\section{Coordination of Kinesin-1 and Dynein on Mitochondria}

The presence of different types of motor proteins on one organelle raises interesting questions about how they are coordinated to accomplish proper cargo transport and distribution (Deacon et al., 2003; Gross, 2003; Welte, 2004). Coordination of cytoplasmic dynein and an unidentified plus-end motor on lipid droplets in Drosophila embryos is sensitive to the dominant-negative $G l^{1}$ allele, implicating the P150Glued component of dynactin in the coordination mechanism (Gross et al., 2002). Our results are consistent with cohabitation of dynein and kinesin-1 on axonal mitochondria and with their activities being coordinated to avoid antagonism, but the coordination mechanism was insensitive to either the $G l^{1}$ allele or neuron-targeted $G l$ RNAi. Both those inhibition approaches enhanced, rather than reduced, plus- and minus-end mitochondrial runs in motor axons. When a more complete disruption of P150Glued in larval neurons becomes possible, perhaps a role in the coordination mechanism will become evident. However, our current data suggest that axonal P150Glued slows mitochondrial runs in both directions. It is an elongated protein with a microtubule-binding site at one end that is thought to facilitate dynein processivity by acting as a tether to hold the dynein and its attached cargo near the microtubule (reviewed by Schroer, 2004). Because mitochondria are sufficiently large to accommodate many motor complexes and they are confined near microtubules by the small diameter of axons, processivity in axons may not need assistance from P150Glued. In the confines of the axon, perhaps the microtubule-binding action of P150Glued actually generates drag that retards rather than facilitates mitochondrial runs.

\section{Directional Programming}

Our results are consistent with a model in which directional programming is controlled by signals that partition mitochondria into distinct anterograde, stationary, and retrograde classes (Hollenbeck, 1996). We suggest that mitochondria with fresh, cell body-derived components generate a local signal that represses cytoplasmic dynein, activates kinesin-1, and thus dictates anterograde transport toward the terminal. In the axon, areas requiring ATP generate local signals that convert anterograde mitochondria to stationary by activating static cross-links with the cytoskeleton (Forman et al., 1987; Chada and Hollenbeck, 2003; Wagner et al., 2003; Miller and Sheetz, 2004; Hollenbeck and Saxton, 2005). Over time, damage to mitochondrial components from reactive oxygen species causes a decline in energy-producing capacity (Hagen et al., 1997) that triggers release from cytoskeletal anchoring, activation of dynein, and repression of kinesin- 1 . After retrograde transport to the cell body, senescent mitochondria are enclosed in membranes by autophagocytosis and then are degraded by fusion with lysosomes (Vargas et al., 1987; Abeliovich and Klionsky, 2001; RodriguezEnriquez et al., 2004). Regulatory mechanisms that control transitions between the three states may be triggered by extracellular signals, local ATP and ion concentrations, mitochondrial inner membrane potential, or other internal cues from mitochondria (Hollenbeck and Saxton, 2005). Definition of those control mechanisms should be an exciting endeavor in the coming years.

\section{Axonal Swellings}

The genesis and nature of axonal swellings is of great interest because they are a prominent pathological feature of Alzheimer's and some motor neuron diseases (Hurd and Saxton, 1996; Stokin et al., 2005). The discovery that kinesin-1 and dynein mutations in Drosophila cause axonal swellings led to the proposal that they are a consequence of traffic jams, i.e., halted organelles impede passage of others causing stochastic pile-ups that locally block transport and force axon swelling (Hurd and Saxton, 1996). However, it is clear from the direct observations presented here that organelles in swellings can be quite mobile and that swellings do not cause a general blockade of mitochondrial transport.

If axonal swellings are not caused by localized transport blockades, what causes them? One answer is suggested by the prominence of large autophagosomes and lysosomal organelles within swellings (Gho et al., 1992; Hurd and Saxton, 1996; Martin et al., 1999a). Mitochondria with damaged proteins and weak membrane potential undergo a membrane permeability transition that allows release of signals for apoptotic and necrotic cell death. That permeability transition also triggers rapid autophagocytosis and lysosomal degradation of mitochondria that presumably help suppress the cell death signaling (reviewed by RodriguezEnriquez et al., 2004). We suggest that mitochondria stranded by failed retrograde transport pass the permeability transition in the axon, release cell death signals and stimulate local axonal autophagocytosis. Structural and physiological changes that accompany the autophagocytosis cause local enlargement of axon diameter. If this hypothesis is true, then the swellings associated with some neurodegenerative diseases may be triggered by failed retrograde transport and the lingering presence of spent mitochondria in axons.

Support for this stranded mitochondria hypothesis comes from classic studies of physical blockades of axonal transport. Anterograde organelles accumulate on the proximal (cell body) side of a blockade, and retrograde organelles accumulate on the distal side (Smith, 1980; Tsukita and Ishikawa, 1980). Proximal organelles can return to the cell body by switching to retrograde transport (Smith, 1980), but distal organelles are trapped in the axon. Among the normal-looking organelles on the distal side, many autophagosomes and lysosomal organelles accumulate (Smith, 1980; Tsukita and Ishikawa, 1980; Hirokawa et al., 1991). The source of those organelles has been puzzling, because they are not common in normal axons. As proposed for autophagocytic/lysosomal organelles in the axonal swellings of Drosophila kinesin-1 and dynein mutants, those distal blockade-induced organelles could reflect local autophagy induced by trapped mitochondria that have passed the membrane permeability transition. Further study of the relationship between mitochondrial transport behavior, physiology, and autophagy may provide new insight into the genesis of axonal swellings and contribute to our understanding of the pathology of neurodegenerative diseases.

\section{ACKNOWLEDGMENTS}

We thank James Powers for constructing the RNAi P-element; Jon Scholey and Rahul Warrior for sharing antibodies used in this study; and Peter Hollenbeck, Volodya Gelfand, Steve Gross, and Thom Kaufman for discussions of the work. This work was supported by National Institutes of Health 
Grant GM-46295 (to W.M.S.), an Established Investigatorship from the American Heart Association (to W.M.S.), and fellowships (to A.D.P. and D.H.) from the American Heart Association, Midwest Affiliate.

\section{REFERENCES}

Abeliovich, H., and Klionsky, D. J. (2001). Autophagy in yeast: mechanistic insights and physiological function. Microbiol. Mol. Biol. Rev. 65, 463-479.

Bowman, A. B., Patel-King, R. S., Benashski, S. E., McCaffery, J. M., Goldstein, L.S.B., and King, S. M. (1999). Drosophila roadblock and Chlamydomonas LC7: a conserved family of dynein-associated proteins involved in axonal transport, flagellar motility, and mitosis. J. Cell Biol. 146, 165-180.

Brady, S. T., Pfister, K. K., and Bloom, G. S. (1990). A monoclonal antibody against kinesin inhibits both anterograde and retrograde fast axonal transport in squid axoplasm. Proc. Natl. Acad. Sci. USA 87, 1061-1065.

Brand, A. H., and Perrimon, N. (1993). Targeted gene expression as a means of altering cell fates and generating dominant phenotypes. Development 118, 401-415.

Brendza, K. M., Rose, D. J., Gilbert, S. P., and Saxton, W. M. (1999). Lethal kinesin mutations reveal amino acids important for ATPase activation and structural coupling. J. Biol. Chem. 274, 31506-31514.

Brendza, R. P., Serbus, L. R., Saxton, W. M., and Duffy, J. B. (2002). Posterior localization of dynein and dorsal-ventral axis formation depends on kinesin in Drosophila oocytes. Curr. Biol. 12, 1541-1545.

Chada, S. R., and Hollenbeck, P. J. (2003). Mitochondrial movement and positioning in axons: the role of growth factor signaling. J. Exp. Biol. 206, 1985-1992.

Chada, S. R., and Hollenbeck, P. J. (2004). Nerve growth factor signaling regulates motility and docking of axonal mitochondria. Curr. Biol. 14, 1272 1276.

Davis, A. F., and Clayton, D. A. (1996). In situ localization of mitochondrial DNA replication in intact mammalian cells. J. Cell Biol. 135, 883-893.

Deacon, S. W., Serpinskaya, A. S., Vaughan, P. S., Lopez Fanarraga, M., Vernos, I., Vaughan, K. T., and Gelfand, V. I. (2003). Dynactin is required for bidirectional organelle transport. J. Cell Biol. 160, 297-301.

Duncan, J. E., and Warrior, R. (2002). The cytoplasmic dynein and kinesin motors have interdependent roles in patterning the Drosophila oocyte. Curr. Biol. 12, 1982-1991.

Ekstrom, P., and Kanje, M. (1984). Inhibition of fast axonal transport by erythro-9-[3-(2-hydroxynonyl)]adenine. J. Neurochem. 43, 1342-1345.

Fan, S. S., and Ready, D. F. (1997). Glued participates in distinct microtubulebased activities in Drosophila eye development. Development 124, 1497-1507.

Forman, D. S., Lynch, K. J., and Smith, R. S. (1987). Organelle dynamics in lobster axons: anterograde, retrograde and stationary mitochondria. Brain Res. 412, 96-106.

Fuchs, F., and Westermann, B. (2005). Role of Unc104/KIF1-related motor proteins in mitochondrial transport in Neurospora crassa. Mol. Biol. Cell 16, 153-161.

Gepner, J., Li, M., Ludmann, S., Kortas, C., Boylan, K., Iyadurai, S. J., McGrail M., and Hays, T. S. (1996). Cytoplasmic dynein function is essential in Drosophila melanogaster. Genetics 142, 865-878.

Gho, M., McDonald, K., Ganetzky, B., and Saxton, W. M. (1992). Effects of kinesin mutations on neuronal functions. Science 258, 313-316.

Gross, S. P. (2003). Dynactin: coordinating motors with opposite inclinations. Curr. Biol. 13, R320-R322.

Gross, S. P., Welte, M. A., Block, S. M., and Wieschaus, E. F. (2002). Coordination of opposite-polarity microtubule motors. J. Cell Biol. 156, 715-724.

Guo, X., Macleod, G. T., Wellington, A., Hu, F., Panchumarthi, S., Schoenfield, M., Marin, L., Charlton, M. P., Atwood, H. L., and Zinsmaier, K. E. (2005). The GTPase dMiro is required for axonal transport of mitochondria to Drosophila synapses. Neuron 47, 379-393.

Hagen, T. M., Yowe, D. L., Bartholomew, J. C., Wehr, C. M., Do, K. L., Park, J. Y., and Ames, B. N. (1997). Mitochondrial decay in hepatocytes from old rats: membrane potential declines, heterogeneity and oxidants increase. Proc. Natl. Acad. Sci. USA 94, 3064-3069.

Hirokawa, N., and Takemura, R. (2005). Molecular motors and mechanisms of directional transport in neurons. Nat. Rev. Neurosci. 6, 201-214.

Hirokawa, N., Sato-Yoshitake, R., Kobayashi, N., Pfister, K. K., Bloom, G. S., and Brady, S. T. (1991). Kinesin associates with anterogradely transported membranous organelles in vivo. J. Cell Biol. 114, 295-302.
Hollenbeck, P. J. (1993). Products of endocytosis and autophagy are retrieved from axons by regulated retrograde organelle transport. J. Cell Biol. 121, 305-315.

Hollenbeck, P. J. (1996). The pattern and mechanism of mitochondrial transport in axons. Front. Biosci. 1, d91-d102.

Hollenbeck, P. J., and Saxton, W. M. (2005). The axonal transport of mitochondria. J. Cell Sci. 118, 5411-5419.

Hurd, D. D., and Saxton, W. M. (1996). Kinesin mutations cause motor neuron disease phenotypes by disrupting fast axonal transport in Drosophila. Genetics 144, 1075-1085.

Kanai, Y., Okada, Y., Tanaka, Y., Harada, A., Terada, S., and Hirokawa, N. (2000). KIF5C, a novel neuronal kinesin enriched in motor neurons. J. Neurosci. $20,6374-6384$.

Khodjakov, A., Lizunova, E. M., Minin, A. A., Koonce, M. P., and Gyoeva, F. K. (1998). A specific light chain of kinesin associates with mitochondria in cultured cells. Mol. Biol. Cell 9, 333-343.

Koushika, S. P., Schaefer, A. M., Vincent, R., Willis, J. H., Bowerman, B., and Nonet, M. L. (2004). Mutations in Caenorhabditis elegans cytoplasmic dynein components reveal specificity of neuronal retrograde cargo. J. Neurosci. 24, 3907-3916.

Kural, C., Kim, H., Syed, S., Goshima, G., Gelfand, V. I., and Selvin, P. R. (2005). Kinesin and dynein move a peroxisome in vivo: a tug-of-war or coordinated movement? Science 308, 1469-1472.

Lalli, G., Gschmeissner, S., and Schiavo, G. (2003). Myosin Va and microtubule-based motors are required for fast axonal retrograde transport of tetanus toxin in motor neurons. J. Cell Sci. 116, 4639-4650.

Lee, Y. S., and Carthew, R. W. (2003). Making a better RNAi vector for Drosophila: use of intron spacers. Methods 30, 322-329.

Leopold, P. L., McDowall, A. W., Pfister, K. K., Bloom, G. S., and Brady, S. T. (1992). Association of kinesin with characterized membrane-bounded organelles. Cell Motil. Cytoskeleton 23, 19-33.

Ligon, L. A., Tokito, M., Finkelstein, J. M., Grossman, F. E., and Holzbaur, E. L. (2004). A direct interaction between cytoplasmic dynein and kinesin I may coordinate motor activity. J. Biol. Chem. 279, 19201-19208.

Mandelkow, E., and Mandelkow, E. M. (2002). Kinesin motors and disease. Trends Cell Biol. 12, 585-591.

Martin, M., Iyadurai, S. J., Gassman, A., Gindhart, J. G., Jr., Hays, T. S., and Saxton, W. M. (1999a). Cytoplasmic dynein, the dynactin complex, and kinesin are interdependent and essential for fast axonal transport. Mol. Biol. Cell 10, 3717-3728.

Martin, M. A., Hurd, D. D., and Saxton, W. M. (1999b). Kinesins in the nervous system. Cell Mol. Life Sci. 56, 200-216.

McGrail, M., Gepner, J., Silvanovich, A., Ludmann, S., Serr, M., and Hays, T. S. (1995). Regulation of cytoplasmic dynein function in vivo by the Drosophila Glued complex. J. Cell Biol. 131, 411-425.

Miller, K. E., and Sheetz, M. P. (2004). Axonal mitochondrial transport and potential are correlated. J. Cell Sci. 117, 2791-2804.

Morris, R. L., and Hollenbeck, P. J. (1993). The regulation of bidirectional mitochondrial transport is coordinated with axonal outgrowth. J. Cell Sci. 104, 917-927.

Nangaku, M., Sato-Yoshitake, R., Okada, Y., Noda, Y., Takemura, R., Yamazaki, H., and Hirokawa, N. (1994). KIF1B, a novel microtubule plus end-directed monomeric motor protein for transport of mitochondria. Cell 79, 1209-1220.

Nixon, R. A., and Cataldo, A. M. (1995). The endosomal-lysosomal system of neurons: new roles. Trends Neurosci. 18, 489-496.

Pfister, K. K. (1999). Cytoplasmic dynein and microtubule transport in the axon: the action connection. Mol. Neurobiol. 20, 81-91.

Rizzuto, R., Brini, M., Pizzo, P., Murgia, M., and Pozzan, T. (1995). Chimeric green fluorescent protein as a tool for visualizing subcellular organelles in living cells. Curr. Biol. 5, 635-642.

Rizzuto, R., Nakase, H., Darras, B., Francke, U., Fabrizi, G. M., Mengel, T., Walsh, F., Kadenbach, B., DiMauro, S., and Schon, E. A. (1989). A gene specifying subunit VIII of human cytochrome c oxidase is localized to chromosome 11 and is expressed in both muscle and non-muscle tissues. J. Biol. Chem. 264, 10595-10600.

Rodriguez-Enriquez, S., He, L., and Lemasters, J. J. (2004). Role of mitochondrial permeability transition pores in mitochondrial autophagy. Int. J. Biochem. Cell Biol. 36, 2463-2472. 


\section{A. D. Pilling et al.}

Ruthel, G., and Hollenbeck, P. J. (2003). Response of mitochondrial traffic to axon determination and differential branch growth. J. Neurosci. 23, 86188624 .

Saxton, W. M., Hicks, J., Goldstein, L.S.B., and Raff, E. C. (1991). Kinesin heavy chain is essential for viability and neuromuscular functions in Drosophila, but mutants show no defects in mitosis. Cell 64, 1093-1102.

Schroer, T. A. (2004). Dynactin. Annu. Rev. Cell Dev. Biol. 20, 759-779.

Sharp, D. J., Rogers, G. C., and Scholey, J. M. (2000). Cytoplasmic dynein is required for poleward chromosome movement during mitosis in Drosophila embryos. Nat. Cell Biol. 2, 922-930.

Smith, R. S. (1980). The short term accumulation of axonally transported organelles in the region of localized lesions of single myelinated axons. J. Neurocytol. 9, 39-65.

Stokin, G. B., et al. (2005). Axonopathy and transport deficits early in the pathogenesis of Alzheimer's disease. Science 307, 1282-1288.

Stowers, R. S., Megeath, L. J., Gorska-Andrzejak, J., Meinertzhagen, I. A., and Schwarz, T. L. (2002). Axonal transport of mitochondria to synapses depends on Milton, a novel Drosophila protein. Neuron 36, 1063-1077.

Susalka, S. J., Hancock, W. O., and Pfister, K. K. (2000). Distinct cytoplasmic dynein complexes are transported by different mechanisms in axons. Biochim. Biophys. Acta 1496, 76-88.

Tanaka, Y., Kanai, Y., Okada, Y., Nonaka, S., Takeda, S., Harada, A., and Hirokawa, N. (1998). Targeted disruption of mouse conventional kinesin heavy chain, kif5B, results in abnormal perinuclear clustering of mitochondria. Cell 93, 1147-1158.

Theiss, C., Napirei, M., and Meller, K. (2005). Impairment of anterograde and retrograde neurofilament transport after anti-kinesin and anti-dynein antibody microinjection in chicken dorsal root ganglia. Eur. J. Cell Biol. 84, 29-43.
Tolkovsky, A. M., Xue, L., Fletcher, G. C., and Borutaite, V. (2002). Mitochondrial disappearance from cells: a clue to the role of autophagy in programmed cell death and disease? Biochimie 84, 233-240.

Tsukita, S., and Ishikawa, H. (1980). The movement of membranous organelles in axons. Electron microscopic identification of anterogradely and retrogradely transported organelles. J. Cell Biol. 84, 513-530.

Vargas, J. L., Roche, E., Knecht, E., and Grisolia, S. (1987). Differences in the half-lives of some mitochondrial rat liver enzymes may derive partially from hepatocyte heterogeneity. FEBS Lett. 224, 182-186.

Wagner, O. I., Lifshitz, J., Janmey, P. A., Linden, M., McIntosh, T. K., and Leterrier, J. F. (2003). Mechanisms of mitochondria-neurofilament interactions. J. Neurosci. 23, 9046-9058.

Wang, C., Asai, D. J., and Robinson, K. R. (1995). Retrograde but not anterograde bead movement in intact axons requires dynein. J. Neurobiol. 27, $216-226$.

Welte, M. A. (2004). Bidirectional transport along microtubules. Curr. Biol. 14, R525-R537.

Welte, M. A., Gross, S. P., Postner, M., Block, S. M., and Wieschaus, E. F. (1998). Developmental regulation of vesicle transport in Drosophila embryos: forces and kinetics. Cell 92, 547-557.

Wu, C. F., Sakai, K., Saito, M., and Hotta, Y. (1990). Giant Drosophila neurons differentiated from cytokinesis-arrested embryonic neuroblasts. J. Neurobiol. 21, 499-507.

Yeh, E., Gustafson, K., and Boulianne, G. L. (1995). Green fluorescent protein as a vital marker and reporter of gene expression in Drosophila. Proc. Natl. Acad. Sci. USA 92, 7036-7040. 